\title{
Cranial mesohepatectomy: a challenging parenchyma-preserving operation for colorectal liver metastases
}

\author{
Georgios C. Sotiropoulos • Stephan Timm • \\ Arnold Radtke • Ernesto P. Molmenti • Hauke Lang
}

Accepted: 12 November 2008 / Published online: 26 November 2008

(C) Springer-Verlag 2008

\section{Dear Editor:}

The treatment of colorectal liver metastases has evolved over time. Improvements in chemotherapeutic regimens coupled with a multidisciplinary approach have resulted in a greater number of patients undergoing liver resections and major surgical challenges. Chemotherapeutic injury to the liver mandates preservation of as much parenchymal volume as possible in order to minimize the risk of liver failure. Furthermore, the potential for second and third resections for recurrent disease mandates the maintenance as much as possible of the intrahepatic anatomical relationships. Our present report describes a cranial mesohepatectomy in a case considered to be inoperable by others.

A 57-year-old woman presented to our institution with a colorectal liver metastasis. Approximately 1 year ago, she had undergone a low anterior resection for a nodal negative rectal adenocarcinoma (pT3N0V0L0R0G2). Adjuvant radiochemotherapy according to the MAYO protocol had

G. C. Sotiropoulos $(\bowtie) \cdot$ S. Timm $\cdot$ A. Radtke $\cdot$ H. Lang

Department of General and Abdominal Surgery,

Johannes Gutenberg University Hospital Mainz,

Langenbeckstraße 1,

55131 Mainz, Germany

e-mail: sotiropoulos@ach.klinik.uni-mainz.de

E. P. Molmenti

Department of Surgery, North Shore University Hospital,

Manhasset, NY, USA been initiated but side effects led to its discontinuation after the first cycle. A computed tomography (CT) scan at the time of presentation showed a $19 \mathrm{~cm}$ in diameter central solitary liver lesion infiltrating the right and middle hepatic veins. A right trisectionectomy (resection of segments 4 8) was considered but not pursued due to the high risk of postoperative liver insufficiency (calculated remnant liver volume of $215 \mathrm{ml}$ ). Careful study of CT images showed a right inferior hepatic vein draining part of the lateral sector of the right liver lobe. Virtual postresection reconstruction with computer-assisted three-dimensional CT showed adequate drainage of segments 5 and 6 and partial drainage of segment $4 \mathrm{~b}(379 \mathrm{ml})$ via the right inferior hepatic vein. A cranial mesohepatectomy (segments 4a, 7, and 8) was undertaken. The lesion was resected en bloc with the right and middle hepatic veins. Pathological examination of the specimen showed tumor-free margins (R0 resection). The patient tolerated the operation well, with no signs of liver insufficiency. She was discharged on postoperative day 10 .

Surgical resection of colorectal liver metastases requires precise anatomical study of the individual intrahepatic anatomy. Recent developments in computer-assisted imaging provide improved visualization of the hepatic vascular and segmental anatomy and allow for volumetric calculation. Image-based computer assistance and three-dimensional reconstruction are particularly useful for planning challenging liver resections, such as cranial mesohepatectomy, minimizing the risk of postoperative liver failure. 